\title{
LAS CALERAS, PROVINCIA DE CÓRDOBA, PRIMER YACIMIENTO FOSILÍFERO DE ORIGEN CÁRSTICO DE ARGENTINA
}

\author{
Adán A. TAUBER \\ CRILAR. Mendoza y Entre Ríos, 5301-Anillaco, La Rioja, Argentina \\ Museo de Ciencias Naturales "Bartolomé Mitre". Hipólito Yrigoyen 115, \\ 5000-Córdoba, Argentina
}

Tauber, A.A. 2000. Las Caleras, provincia de Córdoba, primer yacimiento fosilífero de origen cárstico de Argentina. [Las Caleras, Córdoba Province, the first karstic fossil deposit in Argentina.] Revista Española de Paleontología, 15(2), 233-244. ISSN 0213-6937.

\begin{abstract}
A deposit of fossil vertebrates situated in the Calamuchita County (Córdoba Province) is described in a preliminary way. It was been formed on the peaks of the Sierra Blanca, on the eastern slope of the Sierra de los Comechingones as mainly clastic fillings of narrow hollows produced by dissolution of marble of the metamorphic Precambrian basement, in a incipient karstic landscape. The main species registered are: Tupinambis cf. teguixin, Lutreolina sp., Thylophorops chapalmalensis, Doellotatus chapadmalensis, Ringueletia simpsoni, Macroeuphractus outesi, Dankomys aff. simpsoni, Eumysops sp., Lagostomus (Lagostomopsis) sp., Dolichotis sp., Cardiatherium aff. talicei, Tremacyllus impressus, and Xotodon sp. The fossil mammal content lead us infer that: 1) The carrying sediment was deposited during the Pliocene (Chapadmalalan age). 2) The climate used to be warmer and more humid than it is nowadays. 3) The environment was mainly an open plain with pastures. 4) The development of karst was produced during the Pliocene and thus the metamorphic basement of Sierra Blanca could have been uplifted as a block by at least two tectonic phases in the Neogene. The early phase probably ocurred during Miocene-Pliocene and the other during the Late Pliocene.
\end{abstract}

Keywords: Karst filling, Vertebrate, Pliocene, Argentina.

\section{RESUMEN}

Se describe en forma preliminar un yacimiento fosilífero ubicado en Las Caleras, departamento Calamuchita, provincia de Córdoba, el cual se ha desarrollado en la parte cuspidal de la sierra Blanca, sobre la vertiente oriental de la sierra de Los Comechingones. Se trata de rellenos, principalmente clásticos, de estrechas cubetas producidas por disolución de mármoles del basamento metamórfico precámbrico, en un paisaje cárstico incipiente. Las principales especies registradas son: Tupinambis cf. teguixin, Lutreolina sp., Thylophorops chapalmalensis, Doellotatus chapadmalensis, Ringueletia simpsoni, Macroeuphractus outesi, Dankomys aff. simpsoni, Eumysops sp., Lagostomus (Lagostomopsis) sp., Dolichotis sp., Cardiatherium aff. talicei, Tremacyllus impressus y Xotodon sp. El contenido de mamíferos fósiles permite inferir que: 1) los sedimentos portadores se depositaron en el Plioceno (Chapadmalalense), 2) el clima era más cálido y húmedo que el actual, 3) el ambiente era predominantemente abierto con pastizales, 4) el proceso de carstificación se produjo durante el Plioceno, y el bloque del basamento metamórfico de la sierra Blanca habría tenido por lo menos dos movimientos tectónicos en el Neógeno, uno en la fase temprana Mioceno-Plioceno y el otro durante el Plioceno Tardío.

Palabras clave: Relleno cárstico, Vertebrados, Plioceno, Argentina.

\section{INTRODUCCIÓN}

Los yacimientos paleontológicos neógenos de la provincia de Córdoba son, en general, escasos, pobres en restos fósiles, están poco estudiados o algunos presentan dificultades insalvables para su estudio. Tal es el caso de los depósitos estudiados por Castellanos (1936, 1944, 1958) en el valle de Los Reartes, departamento
Calamuchita, donde la mayor parte de los afloramientos neógenos se encuentran cubiertos por las aguas del embalse del río Los Molinos (Pasotti, 1961). El estudio de nuevos depósitos contribuirá al mejor conocimiento del marco geológico regional, ya que la formación de los mismos estaría vinculada con otros fenómenos concurrentes, como el vulcanismo del departamento Pocho (Kay y Gordillo, 1994), con la formación de los 


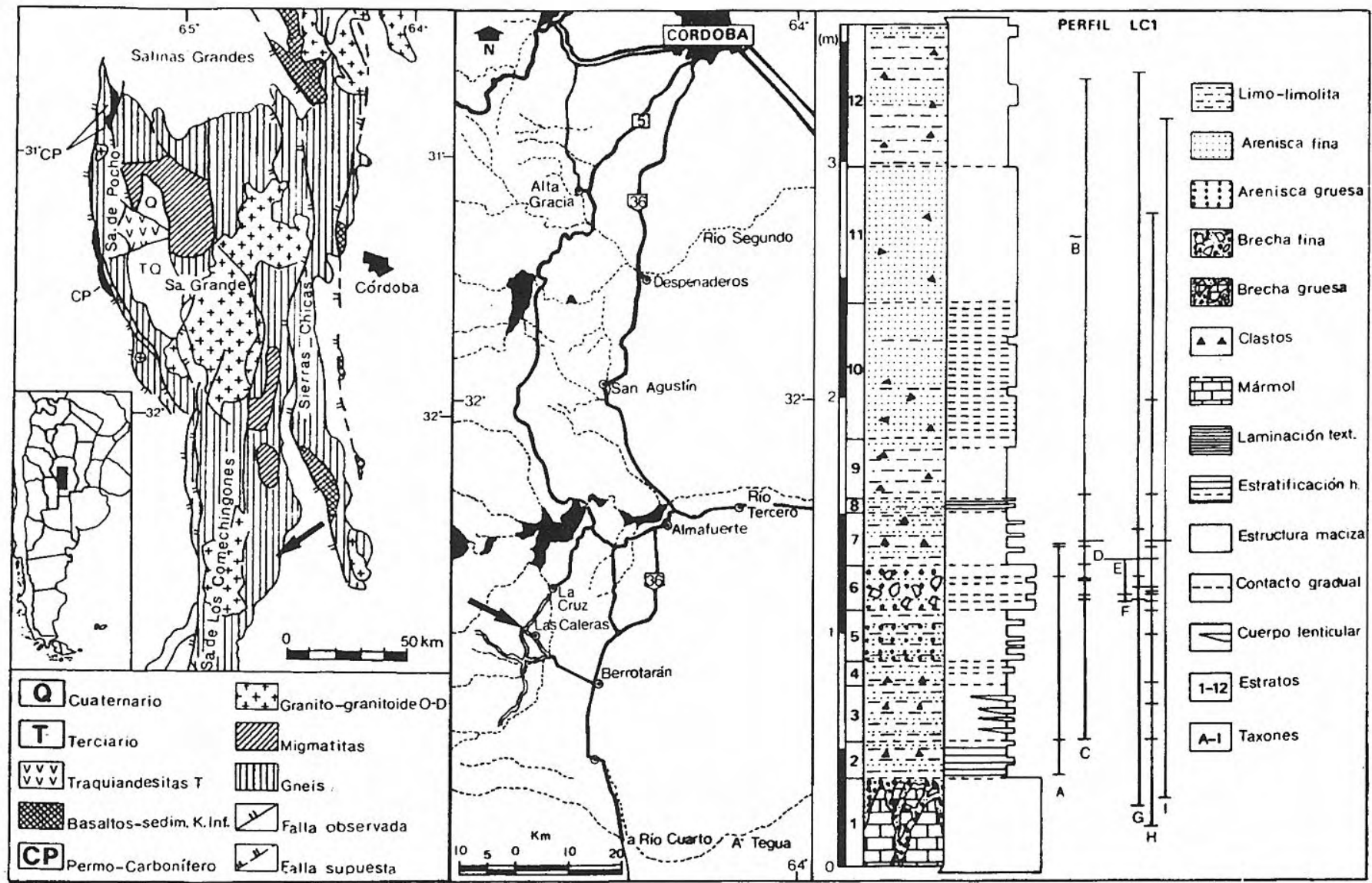

Figura 1. Mapa de ubicación de la localidad fosilífera de Las Caleras y columna estratigráfica del relicto del cuerpo sedimentario Las Caleras 1 (LC1) y su registro paleontológico. A: Lutreolina sp., B: Thylophorops chapalmalensis, C: Doellotatus chapadmalensis, D: Ringueletia simpsoni, E: Dankomys aff. simpsoni, F: Eumysops sp., G: Lagostomus (Lagostomopsis) sp., H: Dolichotis sp., I: Tremacyllus impressus.

Locality map of the Las Caleras fossiliferous site and stratigraphic column with palaeontological record in Las Caleras 1 (LC1).

travertinos de La Playa (Bondesio y Pascual, 1981), con el origen de los grandes valles intermontanos (Sayago, 1979; Kraemer et al., 1993).

La mayoría de los depósitos con vertebrados fósiles del Plioceno y los que se han estudiado más intensamente se hallan restringidos a los afloramientos de la región sur y sureste de la provincia de Buenos Aires, en los acantilados sobre la costa atlántica (Zanchetta, 1995; Cione y Tonni, 1995a, 1995b), con algunas excepciones, como Corral Quemado (Catamarca) (Marshall et al., 1979; Butler et al., 1984). Es necesario el conocimiento de nuevos yacimientos de vertebrados de otras regiones, lo que permitirá confrontar hipótesis y tener una visión más completa sobre aspectos geológicos, climáticos, ambientales, zoogeográficos, taxonómicos y paleobiológicos.

Con el objeto de avanzar en el conocimiento de los eventos geológicos y biológicos producidos durante el Terciario Tardío en las sierras de Córdoba, se describe, en forma preliminar, un nuevo y singular yacimiento de vertebrados fósiles situado en la localidad Las Caleras, pedanía Cañada de Álvarez, departamento Calamuchita, provincia de Córdoba (Tauber et al., 1997b), estando en preparación un análisis sobre aspectos tafonómicos del mismo.

\section{ABREVIATURAS}

LC1, LC2, LC3 y LC4 son los cuerpos sedimentarios de relleno cárstico de Las Caleras. I, C, P y M son los elementos dentarios superiores; i, c, p, y m son los dientes inferiores. CORD-PZ es el acrónimo del Museo de Paleontología de la Universidad Nacional de Córdoba.

\section{ANTECEDENTES Y GENERALIDADES}

El yacimiento fosilífero que se describe está situado en la parte cuspidal de la sierra Blanca, que respresenta las primeras estribaciones sobre la vertiente oriental de la sierra de Los Comechingones, entre Cañada de Álvarez al oeste y el río de La Cruz al este, en el departamento Calamuchita (Fig. 1). En este sector afloran mármoles dolomíticos y calcodolomíticos (Brodtkorb et al., 1981; Martino et al., 1997) portadores de forsterita y diópsido, pertenecientes al basamento metamórfico del Precámbrico Tardío (Linares y La Torre, 1978), intercalados con niveles de gneis biotítico granatífero en parte esquistoso o en parte macizo (Martino et al., 1997). 

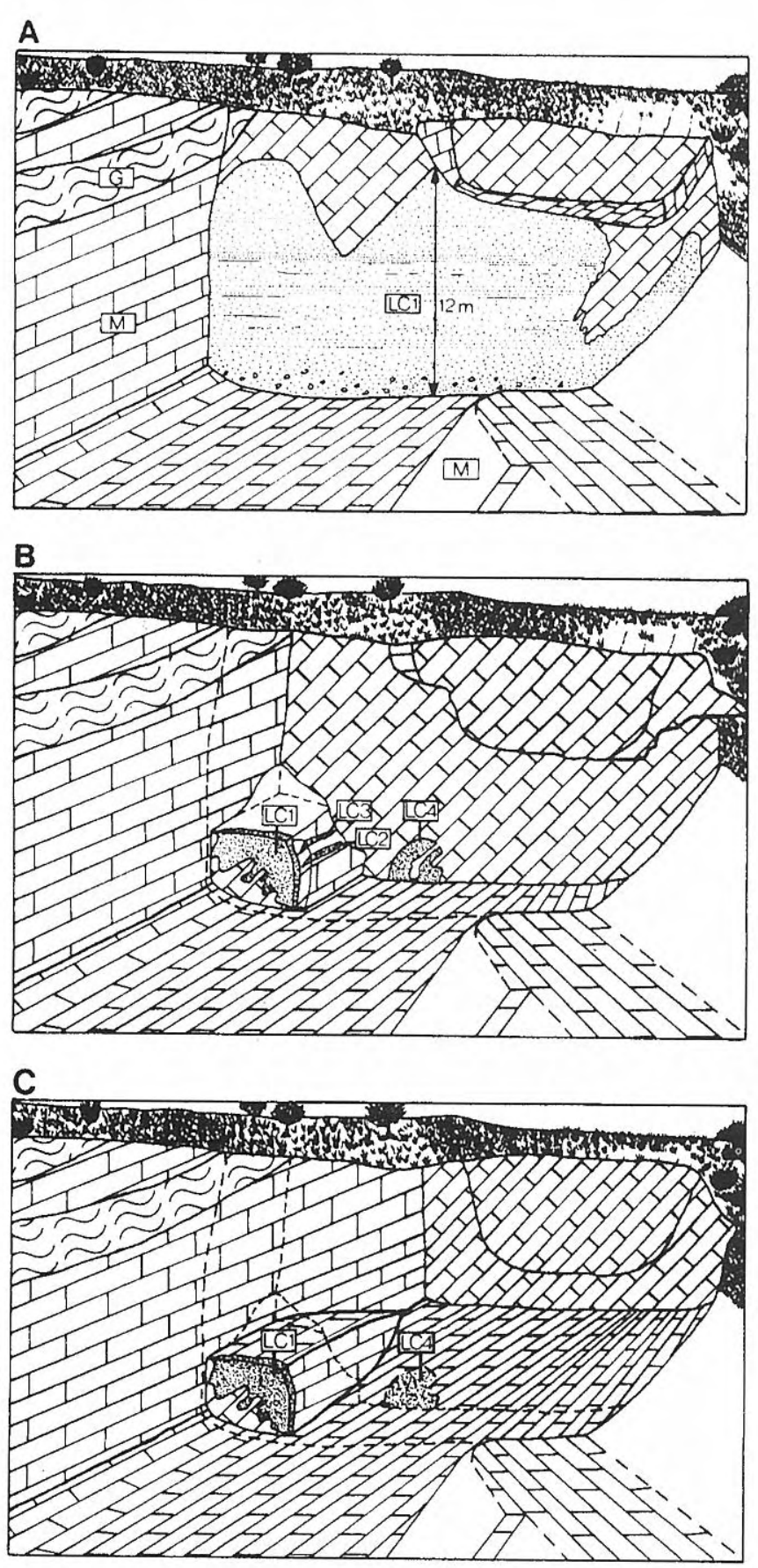

Figura 2. Esquema que muestra el avance de la Cantera Maero. A: modificado de Maero (1994: 55), B: el frente de cantera en diciembre de 1995, C: el frente de cantera en 1996. G: gneis, M: mármol calcodolomítico, LC1-LC4: cuerpos sedimentarios fosilíferos.

Sketch showing the advance of the Maero's quarry. A: modified after Maero (1994: 55), B: the quarry front in December of 1995, C: quarry front in 1996. G: gneiss, M: partly dolomitic marble, LC1-LC4: fossiliferous deposits.

Los afloramientos de mármoles tienen una extensión de $5,15 \mathrm{~km}$ de longitud por $1 \mathrm{~km}$ de anchura, y una dirección predominante de $336^{\circ}$. La megaestructura de las rocas calcáreas y metamórficas intercaladas fue interpretada como un pilar tectónico limitado por fallas, teniendo el conjunto una estructura homoclinal con buzamiento hacia el este (Brodtkorb et al., 1981). Por otro lado, Quintana Salvat (1981) propuso la existencia de un sinclinal volcado. Posteriormente, Martino et al. (1997) interpretaron que la megaestructura es un pliegue sinforme isoclinal volcado con plano axial buzante hacia el este $\left(\mathrm{N} 134^{\circ} / 20^{\circ}\right)$ y eje con dirección e inmersión hacia el sureste (N $134^{\circ} / 24^{\circ}$ ), encontrándose en una cuña de basamento limitada por dos fallas, El Saucesito al este y Viejo Almacén al oeste (Martino et al., 1997).

La cuña de basamento se encuentra elevada tectónicamente, posee una pendiente suave hacia el este y abrupta hacia el oeste, concordando con el estilo general de todas las Sierras Pampeanas de Córdoba. Hacia el sur, su altura desciende gradualmente, encontrándose cubierto por sedimentos del Cenozoico Superior. Los mármoles han tenido una fase de deformación plástica, produciendo pliegues ligeremente volcados hacia el oeste, los cuales pueden observarse en los frentes de cantera, y otra fase de deformación frágil, produciendo varios juegos de diaclasas. En las diaclasas y en menor grado en los planos de estratificación se ha producido el ensanchamiento por un fenómeno de disolución y posterior reprecipitación de la calcita y dolomita, formando un paisaje cárstico incipiente con estructuras típicas, como estalagmitas, estalactitas, columnas de $1,20 \mathrm{~m}$ de altura, estructuras botroidales, aciculares, cortinas, etc.

Los mármoles son explotados con fines industriales para la elaboración de cemento, esto ha producido la exposición de varios cuerpos sedimentarios clásticos con fósiles, y también la destrucción de la mayor parte del yacimiento que se describe aquí. Una gran parte de los bloques con fósiles que fueron removidos se encuentra ahora debajo de la escombrera ( $32^{\circ} 23^{\prime} 30,3^{\prime \prime} \mathrm{S}$ y $\left.64^{\circ} 32^{\prime} 03,1^{\prime \prime} \mathrm{O}\right)$, limitando la calidad de las observaciones e impidiendo conocer la secuencia estratigráfica y bioestratigráfica normal. No obstante, existe un pequeño sector del cuerpo sedimentario principal que se encuentra in situ, el cual fue preservado como testigo.

En cinco sitios alineados con dirección aproximadamente coincidente con el rumbo general de la megaestructura de los mármoles, se observó un sistema de pequeñas galerías, cavernas, simas o torcas (2,5 m de ancho máximo). Estas aberturas se encuentran total o parcialmente rellenadas con material clástico heterométrico. En el sector norte, el material de relleno predominante es limo arcilloso bien seleccionado con laminación paralela o estratificación fina horizontal, y prácticamente no se observaron restos fósiles.

En el sitio $2\left(32^{\circ} 23^{\prime} 05,8^{\prime \prime} \mathrm{S}\right.$ y $\left.64^{\circ} 3156,7^{\prime \prime} \mathrm{O}\right)$ se observaron tres galerías que fueron controladas por un juego de diaclasas. Estas galerías están parcialmente rellenadas con sedimentos cársticos finos, predominantemente limo, los que se hallan cubiertos por una costra calcárea, teniendo ésta un espesor que varía entre 2 y $10 \mathrm{~cm}$ con unos $5 \mathrm{~cm}$ de espesor promedio. En los $10 \mathrm{~cm}$ inferiores a esta costra existen láminas calcáreas con espesores crecientes hacia arriba, intercaladas con limos. En esta costra se encuentran incrustados bloques angulosos de mármoles de hasta $30 \mathrm{~cm}$ 
de diámetro, en posición inestable, producto del desprendimiento del techo de la caverna. En dos de las galerías de este sitio se ha observado el techo abovedado y probablemente la sección de las mismas tendría forma de "ojo de cerradura" (galería paragenética), característica de la zona de circulación vadosa. En estas galerías se observaron estalagmitas, estalactitas, columnas, estructuras botroidales, aciculares, cortinas, etc.

\section{PRINCIPAL CUERPO FOSILÍFERO}

El sitio 5 es el más importante desde el punto de vista paleontológico, está ubicado en la cantera Maero $\left(32^{\circ} 23^{\prime}\right.$ $35,1^{\prime \prime}$ S y $64^{\circ} 31^{\prime} 54,5^{\prime}$ ' O, frente de cantera 3, nivel 2, Fig. 2). Allí se encuentra in situ un relicto del cuerpo sedimentario más rico en restos de vertebrados fósiles, ya que, la mayor parte de este depósito fue destruido por la explotación de los mármoles.

Este cuerpo constituía el relleno de cuatro aberturas principales conectadas entre sí y con diferentes posiciones. Dos de estas aberturas menores se formaron a partir de planos de estratificación del mármol, las cuales estaban rellenas con arena gruesa (LC2, LC3, Fig. 2). La mayor de esas cavidades (LC4) tenía una anchura máxima visible de $3,4 \mathrm{~m}$ y su longitud observada por los obreros de la cantera era de $40 \mathrm{~m}$ aproximadamente. Su dirección coincidía con el rumbo general de los mármoles que en este sitio es de $351^{\circ} \mathrm{y}$ $26^{\circ}$ de buzamiento hacia el este. Esta cavidad fue destruida, en su mayor parte, durante la extracción de estas rocas y antes de ser observada por el autor de este trabajo. Sin embargo, continúa aparentemente por debajo del nivel de explotación 2 de la cantera Maero. El material de relleno está compuesto por brecha maciza, limo laminado y arena gruesa con estratificación fina en posición horizontal, siendo portador de restos de pequeñas aves y mamíferos.

La otra parte del cuerpo sedimentario corresponde al relleno de una diaclasa de rumbo $81^{\circ}$ y buzamiento $71^{\circ}$ hacia el norte (LC1). La anchura del depósito clástico era de 1,70 $\mathrm{m}$ (en dirección norte sur) y $15 \mathrm{~m}$ aproximadamente (en dirección este oeste); el espesor originalmente visible era de 13,5 m (Fig. 2-A y Maero, 1994: 55). La mayor parte de este relleno fue volado durante la explotación de los mármoles en el año 1995; sin embargo, aún se preserva un relicto in situ de $5,5 \times 1,25 \mathrm{~m}$ de superficie y $3,5 \mathrm{~m}$ de espesor y los bloques extraídos se encuentran en la escombrera. El relleno clástico predominante es limo macizo o con estratificación pobremente definida; en algunos sectores se encuentra fuertemente cementado con carbonato de calcio. Existen además intercalaciones subordinadas de arenas y areniscas gruesas en estratos de $1 \mathrm{~cm}$ de espesor. La fracción gruesa predomina en la base del relleno, donde hay bloques angulosos de tamaños variados entre 10 y $30 \mathrm{~cm}$ de diámetro máximo, incluidos en una matriz arenosa maciza. Hacia arriba hay pocos niveles que incluyen clastos angulosos de 1 a $5 \mathrm{~cm}$ de diámetro máximo, soportados por la matriz, que muestran una estratificación paralela poco definida, teniendo una posición prácticamente horizontal (rumbo $40^{\circ}$, dirección de buzamiento $310^{\circ}$ y $1^{\circ}$ de inclinación).

\section{PALEONTOLOGÍA}

En la colección de fósiles recuperados hasta el presente (un millar de huesos aproximadamente), se hallan representados unos 29 taxones principalmente referibles a mamíferos, aunque también se registraron gasterópodos, anuros, reptiles y aves, de los cuales se presentan sólo 13 en este primer trabajo preliminar. Se trata del yacimiento con mayor diversidad específica, cantidad y concentración de restos óseos que se haya registrado hasta el presente en la provincia de Córdoba, habiéndose contabilizado hasta 25 huesos por $\mathrm{kg}$ de sedimento (Tauber et al., 1997a). Los elementos empleados en este estudio se hallan depositados en el Museo de Paleontología, Facultad de Ciencias Exactas, Físicas y Naturales, de la Universidad Nacional de Córdoba, cuyo acrónimo es CORD-PZ; los números de colección son los que figuran entre corchetes.

ORDEN SQUAMATA Merren, 1820 SUBORDEN SCIONCOMORPHA Camp, 1923 Familia Teiidae Gray, 1827

Figura 3. Algunos de los restos de vertebrados registrados en el yacimiento de Las Caleras. a Tupinambis cf. teguixin Linneo (CORD-PZ 4170-1). Dentario derecho con 8 dientes en vista lateral externa. b Lutreolina sp. (CORD-PZ 4003). Dentario izquierdo con la serie p3-m4 en vista externa. c Thylophorops chapalmalensis Ameghino (CORD-PZ 4110). Dentario izquierdo con las raíces del p1, y la serie p2-m4 en vista interna. d Doellotatus chapadmalensis Bordas (CORD-PZ 4026). Placa de una banda movible en vista externa. e Ringueletia simpsoni (Bordas) (CORD-PZ 4002-1). Placa de una banda movible en vista externa. f Macroeuphractus outesi Ameghino (CORD-PZ 4001-4). Placa de una banda movible en vista externa. g Eumysops sp. (CORD-PZ 4004-23). Dentario derecho con il y la serie m1-m3 en vista oclusal. h Lagostomus (Lagostomopsis) sp. Ameghino (CORD-PZ 4057). Mandíbula con los i1, p4, m1, m2 y parte de los $\mathrm{m} 3$ de ambos lados en vista oclusal. i Dolichotis sp. (CORD-PZ 4111). Rama mandibular izquierda con i1 y la serie m1-m3 en vista lateral externa. j Cardiatherium aff. talicei Francis y Mones (CORD-PZ 4090). Fragmento anterior del dentario izquierdo con $\mathrm{i} 1, \mathrm{p} 4, \mathrm{~m} 1$ y parte del $\mathrm{m} 2$ en vista oclusal. k Tremacyllus impressus Ameghino (CORD-PZ 4112-2). Mitad anterior de cráneo con los I1 y las series P2-M3 de ambos lados en vista ventral. 1 Xotodon sp. (CORD-PZ 4019-5). Mitad anterior de un premolar superior izquierdo en vista oclusal. Escala $1 \mathrm{~cm}$.

Some fossil bones of the Las Caleras deposit. a Right dentary with 8 teeth in external view. b Left dentary with p3$m 4$ in external view. c Left dentary with p2-m4 in external view. d External view of a plate of a mobile zone. $\boldsymbol{e}$ External view of a plate of a mobile zone. $f$ External view of a plate of a mobile zone. $\mathrm{g}$ Right dentary with il, ml-m3 in occlusal view. $\mathbf{h}$ Mandible with il, $p 4-m 2$ and fragment of $m 3$ of both side in oclusal view. $\mathbf{i}$ Left dentary with il, $m 1-m 3$ in external view. $\mathbf{j}$ Left dentary fragment with i1, p4-m2 in occlusal view. $\mathbf{k}$ Palatal fragment with I1, P2-M3 of both sides in ventral view. 1 Anterior half of a premolar in occlusal view. Scale bar equals $1 \mathrm{~cm}$. 
a
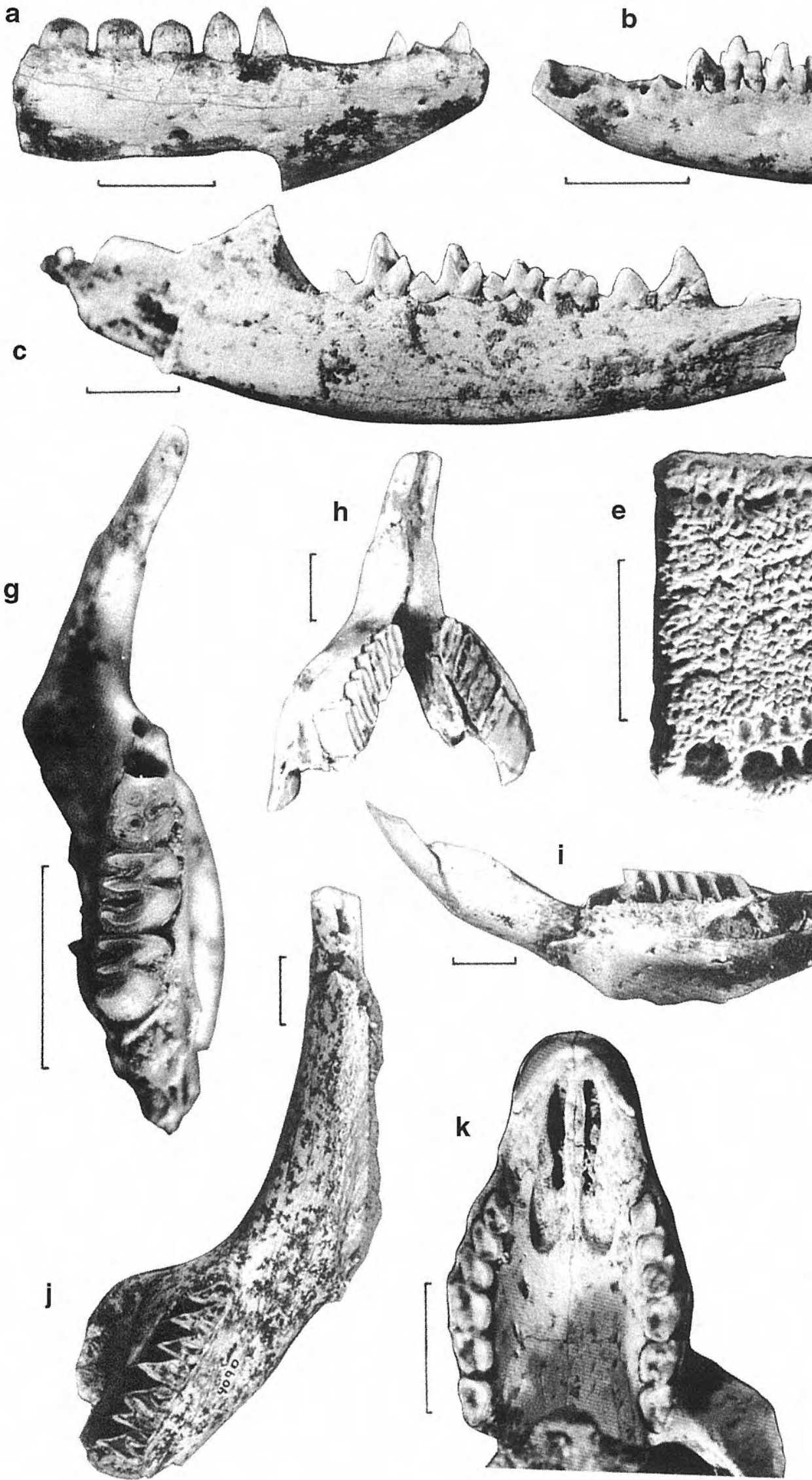

b
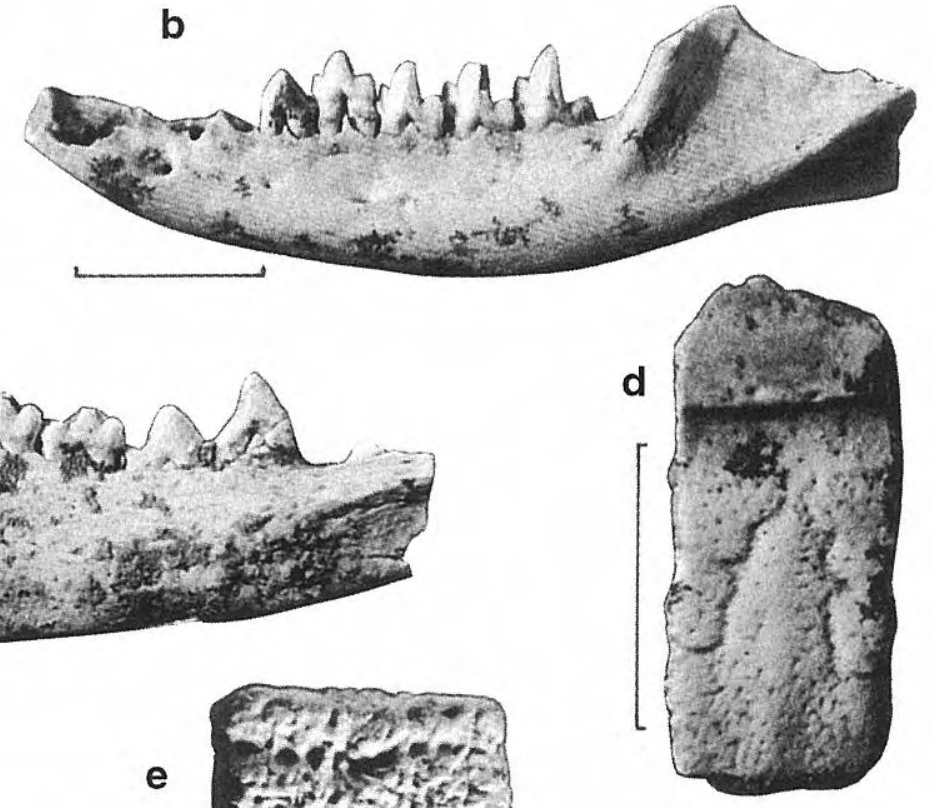

f

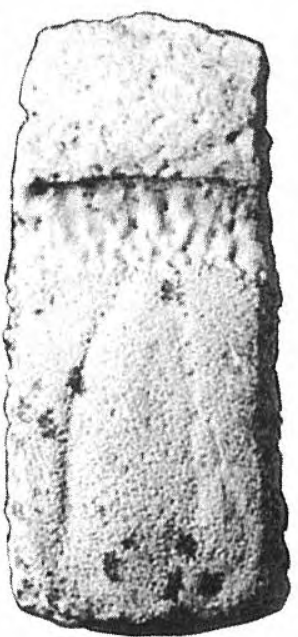

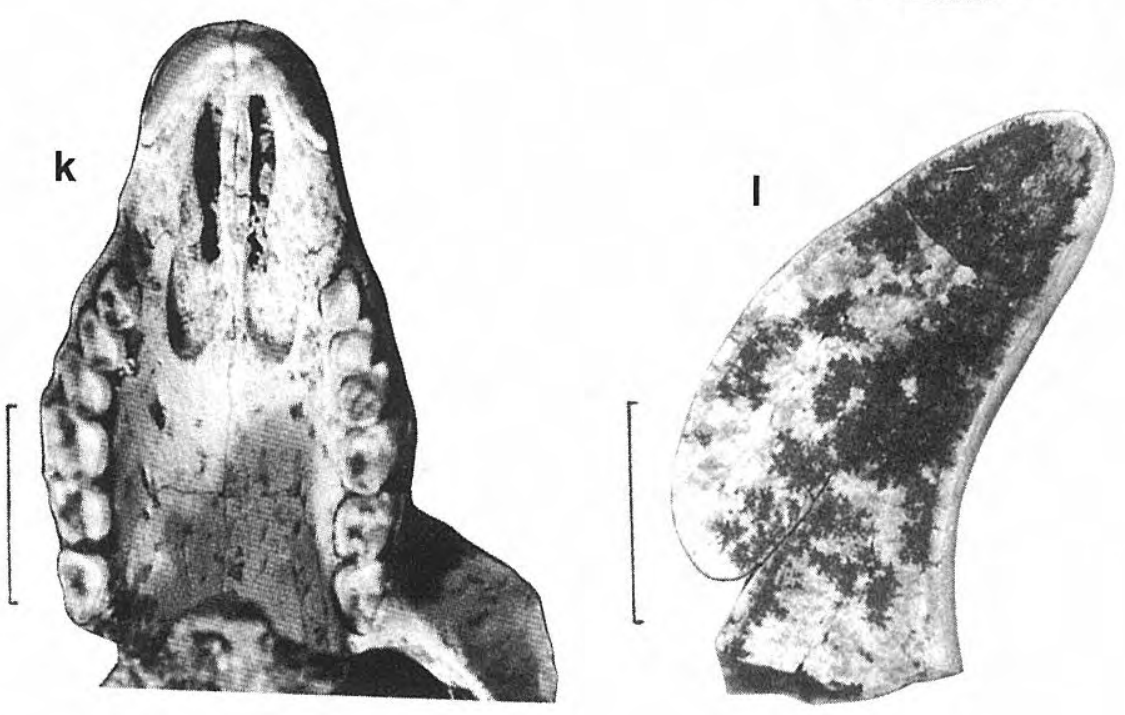


Subfamilia Tupinambinae Presch, 1974

Género Tupinambis Daudin, 1802

\section{Tupinambis cf. teguixin Linneo, 1766}

Fig. 3a

\section{Material y procedencia}

Dentario derecho con 8 dientes [4170-1], dentario izquierdo con 3 dientes y partes de otros dientes [4170-2], premaxilar izquierdo con 4 dientes [4170-3], mitad proximal del cúbito izquierdo [4170-4]. El material procede del bloque S10, escombrera de la cantera Maero.

\section{Comentario}

Algunos rasgos que distinguen las dos especies que actualmente habitan en Argentina, $T$. rufescens (Günther) y $T$. teguixin, son que la primera es de gran tamaño, robusta, de cráneo ancho y tiene extremidades cortas (Cei, 1993). El tamaño del fragmento de húmero en proporción a los maxilares y dentario podría indicar que se trataba de T. teguixin, sin embargo, son necesarias más evidencias para realizar una determinación más precisa.

CLASE MAMMALIA Linneo, 1758

SUPERCOHORTE MARSUPIALIA (Illiger, 1811)

ORDEN DIDELPHIMORPHIA (Gill, 1872) Aplin y Archer, 1987

Familia Didelphidae Gray, 1821

Subfamilia Didelphinae Gray, 1821

Tribu Didelphini Reig, 1981

Género Lutreolina Thomas, 1910

\section{Lutreolina sp.}

Fig. 3b

\section{Material y procedencia}

Dentario izquierdo con c-m2 [4067, LC1], c derecho [4053-5, LC1], dentario derecho con c-m4 [4064-3, LC1], maxilar izquierdo con P3-M3 [4064-4, LC1], maxilar izquierdo con P3-M2 [4064-5, LC1], dentario izquierdo con p3-m2 [4058-1, LC1], dentario izquierdo con la serie p3-m4 [4003].

\section{Comentario}

Estos restos fósiles muestran cierta afinidad con $L$. tracheia Rovereto, 1914; sin embargo, la cantidad de material no permite por el momento una asignación taxonómica precisa en el ámbito específico.

Género Thylophorops Reig, 1952

Thylophorops chapalmalensis (Ameghino, 1908)

Fig. 3c

\section{Material y procedencia}

Dentario izquierdo con p2-m4 y las raíces del p1 [4110,

\section{Comentario}

Esta rama mandibular fue comparada con el neotipo, siendo ambos especímenes prácticamente indiferenciables.

\section{SUPERORDEN XENARTHRA Cope, 1889 \\ ORDEN CINGULATA Illiger, 1811}

Familia Dasypodidae Bonaparte, 1838

Subfamilia Euphractinae Pocock, 1924

Tribu Eutatini Bordas, 1933

Género Doellotatus Bordas, 1932

Doellotatus chapadmalensis Bordas, 1933

Fig. 3d

\section{Material y procedencia}

Placas de las bandas movibles [4026, LC1; 4053-8, LC1; 4025-2, LC1; 4024-2, LC1], placas de las bandas movibles y centro vertebral [4043-4/6, LC1], placas del escudo pélvico [4024-1, LC1; 4033-3, LC1] y una vértebra caudal [4033-2, LC1], 1 falange, 2 placas de las bandas movibles y 1 del escudo pélvico [4053-6/9, LC1]. Otros restos pertenecen probablemente a la especie $[4100, \mathrm{LC} 2,4005-1 / 2, \mathrm{LC} 1]$.

\section{Comentario}

La asignación específica de estos materiales se realizó fundamentalmente por la forma del dibujo central de las placas movibles, que tienen una forma ovalada y relieve muy suave, y presentan un número variable de orificios muy pequeños.

Género Ringueletia Reig, 1958

Ringueletia simpsoni (Bordas, 1933)

Fig. $3 \mathrm{e}$

\section{Material y procedencia}

Placas de las bandas movibles [4002-1, LC4; 4002-2, LC4], placa del escudo pélvico [4106-1, LC2], 1 placa del escudo pélvico y falange [4096-1/2, LC2], calcáneo izquierdo [4002-3, LC4], astrágalo [4057-1, LC1].

\section{Comentario}

Esta es la única especie que se conoce del género; tiene una forma muy característica. La determinación sistemática se realizó teniendo en cuenta la forma de las placas óseas; estas tienen una superficie externa rugosa con el relieve en las figuras muy pobremente definido; tienen doble hilera de orificios pilíferos cercanos al borde posterior de la placa, siendo los posteriores los de mayor diámetro.

Tribu Euphractini Pocock, 1924 Género Macroeuphractus Ameghino, 1887

Macroeuphractus outesi Ameghino, 1908 Fig. $3 \mathrm{f}$ 


\section{Material y procedencia}

5 placas de bandas movibles [4001-4, LC4; 4004-10, LC4; 4001-12, LC4; 4019-2, 4087-1], placa del borde del caparazón [4001-13, LC4], placa del escudo cefálico [40019, LC4], 3 molariformes [4001-5, LC4; 4001-6, LC4; 40018, LC4], fragmentos mandibulares [4001-7, LC4; 4001-22, LC4; 4001-23, LC4], 1 metacarpo? (II?) [4001-10, LC4], 1 falange media? (dígito IV? izquierdo?) [4004-12, LC4], placa del borde del caparazón [4001-14, LC4], placas del escudo pélvico [4019-1, 4019-3], porción de carpo o tarso [4004-11, LC4], 1 metápodo [4001-15, LC4].

\section{Comentario}

Este material es asignado a $M$. outesi siguiendo el criterio de Scillato Yané (1975). Esta especie se distingue de M. morenoi (Lydekker) y M. retusus Ameghino por su gran tamaño y por el mayor ensanchamiento posterior de la figura central.

ORDEN RODENTIA Bowdich, 1821

SUBORDEN MYOMORPHA Brandt, 1877

Superfamilia Muroidea Miller y Gidley, 1918

Familia Cricetidae Rochebrune, 1883

Subfamilia Sigmodontinae Thomas, 1897

Tribu Akodontini Vorontzov, 1959

\section{Dankomys aff. simpsoni Reig, 1978}

Fig. 4

\section{Material y procedencia}

Dentario izquierdo con i, m1-m3 [4040, LC1].

\section{Comentario}

El espécimen estudiado muestra signos de un desgaste avanzado; sin embargo, se observan algunos caracteres que podrían ser intermedios entre $D$. simpsoni y $D$. vorohuensis Reig, 1994. El posterofléxido está poco desarrollado en el m1 y sobre todo en m2. Además, el protofléxido está ausente o poco desarrollado en $D$. vorohuensis y en el espécimen estudiado falta, pero estos rasgos podrían deberse al intenso desgaste. La mayor oblicuidad del posterolofo del $\mathrm{m} 2$ muestra una mayor semejanza con $D$. vorohuensis, aunque esto no es tan claro en el entolófido del mismo diente, y en el $\mathrm{m} 1$ ambos lofos se disponen como en D. simpsoni. La presencia de un fléxido ánteromediano es característica de $D$. simpsoni y en el espécimen estudiado es bien visible, por lo que se considera tentativamente afín a esta última especie, hasta contar con más material. En el $\mathrm{m} 3$ está presente un hipofléxido muy profundo y no se observan vestigios de los fléxidos en la cara lingual (mesofléxido o posterofléxido); este rasgo permite inferir que podría tratarse de un taxón diferente a los anteriormente citados; sin embargo, su establecimiento requeriría el estudio de más material.

\author{
Superfamilia Octodontoidea Simpson, 1945 \\ Familia Echimyidae Gray, 1825 \\ Subfamilia Eumysopinae Rusconi, 1935 \\ Género Eumysops Ameghino, 1888
}

\section{Eumysops sp. \\ Fig. $3 g$}

\section{Material y procedencia}

Dentario derecho con i1 y p4-m3 [4051-7, LC1], dentario derecho con i1, p4-m1 [4002-4, LC4], dentario derecho con i1, m1-m2 [4002-5, LC4], cúbito izquierdo [4002-6, LC4], m1-m3 derechos [4004-23, LC4], dentario derecho con i1, m1-m2 [4002-5, LC4], cúbito izquierdo [4002-6, LC4], m1-m3 derechos [4004-23, LC4], p4-m3 derechos [4004-24, LC4], p4-m2 izquierdos [4044-1, LC1], dentario izquierdo con i1 y m3 [4004-20, LC4], dentario derecho con p4-m2, y m3 aislado [4025-6/11, LC1].

\section{Comentario}

La taxonomía de este género es bastante confusa. Kraglievich $(1957,1965)$ y Vucetich y Verzi (1995) propusieron un esquema sistemático, sin embargo, es necesario realizar una revisión profunda de las especies de Eumysops, tarea que escapa a los objetivos de este trabajo. En los restos hallados en Las Caleras hay una marcada diversidad de formas y tamaños, por lo probablemente estén representadas más de una especie.

Superfamilia Chinchilloidea Kraglievich, 1940 Familia Chinchillidae Bennet, 1833 Género Lagostomus Brookes, 1828

\section{Lagostomus (Lagostomopsis) sp.} Fig. 3h

\section{Material y procedencia}

Maxilar izquierdo con P4-M3 [4060-1, LC1], 2 astrágalos izquierdos [4060-2, LC1, 4107-2], calcáneo izquierdo [4062-3], 1 metápodo y 2 falanges [4060-3/4, LC1], 2 molares [4044-2, LC1], dentario izquierdo con i1, p4-m2 [4014-1], fragmento de molar [4107-1], dentario izquierdo con i1, m1-m2 [4004-1, LC4], dentario derecho con i1-m3 y 1 falange [4035-1/2, LC1], 1 molar inferior [4058-3, LC1], dentario izquierdo con i1, p4-m3 [4033-1, LC1], mandíbula con i1-m3 de ambos lados [4057-2, LC1], 3 molares superiores y M3 izquierdo [4065, LC1].

\section{Comentario}

Kraglievich (1934) reconoce Lagostomopsis como un género diferente de Lagostomus, mientras que Francis y Mones (1966) lo consideran como un subgénero del segundo. Las diferencias entre ambos taxones se refieren esencialmente a la talla y a las distintas proporciones craneanas y dentales, por lo que aquí se incluye Lagostomopsis dentro del género Lagostomus, coincidiendo con Francis y Mones (1966) y con Vucetich y Verzi (1995). Los materiales hallados no permiten una determinación específica. 


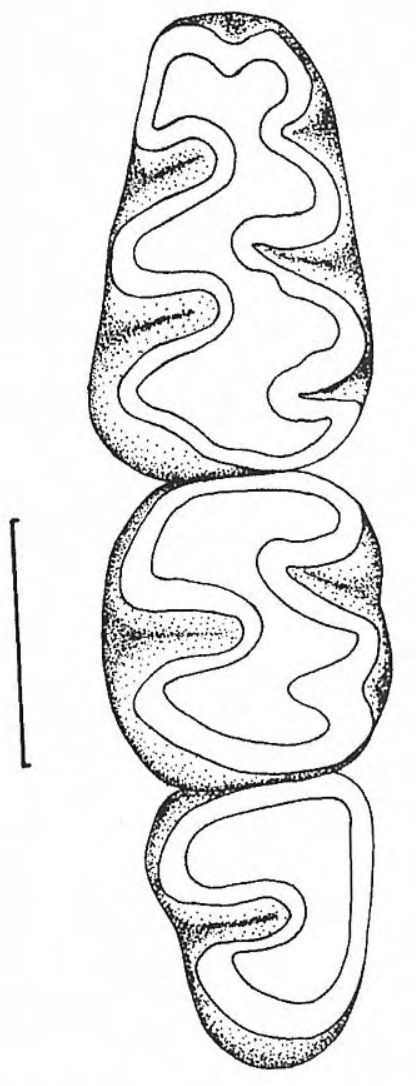

Figura 4. Dankomys aff. simpsoni Reig (CORD-PZ 4040). Molares inferiores en vista oclusal. Escala $1 \mathrm{~mm}$. Lower molar teeth in occlusal view. Scale bar equals $1 \mathrm{~mm}$.

\section{Superfamilia Cavioidea Kraglievich, 1930 \\ Familia Caviidae Gray, 1821 \\ Subfamilia Dolichotinae Pocock, 1922 \\ Género Dolichotis Desmarest, 1820}

\section{Dolichotis sp.}

Fig. $3 i$

\section{Material y procedencia}

Dentario izquierdo con i1, p4-m3 [4005-3, LC1], tibias izquierdas [4105-1, LC2; 4072, LC1], parte del fémur y calcáneo derechos [4093-3/4, LC2], dentario izquierdo con i1, p4-m2 [4076, LC1], astrágalo izquierdo [4077-9, LC1], parte de un molar o premolar [4077-8, LC1], fragmento de coxal [4077-1, LC1], extremo proximal del radio izquierdo [4077-5, LC1], metápodo [4077-12, LC1], parte de la mandíbula con i1 [4077-13, LC1], fragmento de maxilar izquierdo [4083-1, LC1], 7 molares [4083-2/6, LC1; 4055-2, LC1; 4064-2, LC1], calcáneos izquierdos [4083-7, LC1; 4006-2], dentario derecho con p4-m3 [4025-1, LC1], dentario izquierdo con p4-m3 [4068, LC1], p4 izquierdo [4055-1, LC1], falanges [4055-3/4, LC1; 4045-4, LC1], M3 derecho [4037-2, LC1], fragmento de metápodo [4037-1, LC1], mitad proximal del metatarso III derecho [4051-2, LC1], p4 izquierdo [4051-4, LC1], trozos de incisivos [4064-1,LC1; 4045-1/3, LC1; 4053-2, LC1], parte del premaxilar derecho [4045-5, LC1], astrágalos derechos
[4099-1/2, LC2], p4 izquierdo [4096-2, LC2], m2? derecho [4027, LC1], extremo proximal de tibia derecha [4006-1, LC1], extremo distal de tibia derecha [4006-3, LC1], astrágalo izquierdo [4006-4, LC1], 2 metatarsos III derechos [4006-5/6, LC1], i1 izquierdo [4006-4, LC1], 2 metatarsos III derechos [4006-5/6, LC1], i1 izquierdo [4003-2, LC4], fémur derecho [4071, LC1], mitad distal de la tibia izquierda [4077-6, LC1], cúbito izquierdo [4053-1, LC1], porción de metápodo [4053-1, LC1], porción de metápodo [4053-4, LC1], dentario, i1 y p4 derecho [4077-14, LC1], dentario izquierdo con i1, m1-m3 [4111, LC1], trozo de cráneo con P4-M1 izquierdo y P4-M3 derechos [4006-7, LC1].

\section{Comentario}

Estos materiales son asignados a Dolichotis por tener proporciones similares a las de la especie actual $D$. patagonum (Zimmerman) (es decir que tienen un rostro largo), y por tener el prisma posterior del m3 bien desarrollado y delimitado por un surco estrecho y profundo en la cara lingual. Sin embargo, difieren de dicha especie por el tamaño de todos los especímenes, que es considerablemente menor y similar al de $D$. chapadmalensis Ameghino. Una determinación a nivel específico requiere una revisión detallada.

Familia Hydrochoeridae Gill, 1872

Subfamilia Cardiatherinae Kraglievich, 1930 Género Cardiatherium Ameghino, 1883

\section{Cardiatherium aff. talicei Francis y Mones, 1965}

Fig. 3j

\section{Material y procedencia}

Rama mandibular izquierda con i1, p4-m2 [4090], rama madibular izquierda con p4-m2 [4089]. 2 incisivos [4001-2, LC4; 4001-21, LC4] pertenecen probablemente a esta especie.

\section{Comentario}

Los especímenes CORD-PZ 4090 y 4089 muestran caracteres similares y en parte intermedios a aquellos de C. isseli Rovereto, 1914 y C. talicei; no obstante, existen ligeras diferencias con ambas especies. En efecto, los restos aquí estudiados se asemejan a $C$. isseli por poseer hendiduras interprismáticas más profundas que en $C$. talicei; además, tienen en el p4 una h5i (hti) bien marcada y situada delante de la h2e, a diferencia de $C$. talicei, que tiene una h5i (hti) moderadamente marcada y enfrentada a la h2e (ver nomenclatura en Mones, 1991). Sin embargo, este último rasgo podría ser una variación intraespecífica, teniendo en cuenta la variabilidad morfológica señalada por Mones (1991) en especímenes de Hydrochoerus hydrochaeris (Linneo). Por otro lado, la h2i se encuentra delante de h1e, como en el holotipo de $C$. isseli, y no enfrentada con hle, como sucede en $C$. talice $i$; pero esto también podría ser una variación intraespecífica (ver $C$. aff. isseli en Pascual y Bondesio, 1985; Mones 1991). 
Otros dos caracteres permiten distinguir $C$. isseli de $C$. talicei. Uno es la anchura proporcionalmente mayor en los molariformes inferiores de la última especie y en este rasgo los restos de Las Caleras son similares a $C$. talicei. El otro carácter es que las hendiduras son más anchas en C. talicei y en CORD-PZ 4089 y 4090 que en C. isseli. El tamaño de todos los especímenes de Las Caleras es considerablemente menor que el de las otras dos especies comparadas, sobre todo es más marcada la diferencia con C. isseli.

ORDEN NOTOUNGULATA Roth, 1903 SUBORDEN HEGETOTHERIA Simpson, 1945

Familia Hegetotheriidae Ameghino, 1894

Género Tremacyllus Ameghino, 1891

\section{Tremacyllus impressus (Ameghino, 1888) Fig. 3k}

\begin{abstract}
Material y procedencia
M1-M2 [4102, LC2], maxilares y premaxilares con I1, y la serie P2-M3 derecha e I1, P2-M1 izquierdos [4010], mandíbula con i1-i2 y p2-m3 izquierda, i1, i2, p2 y p3 derechos [4066, LC1], mandíbula con i1-m2 derechos y p4$\mathrm{m} 2$ izquierdos [4112-1, LC1], cráneo con todos los dientes [4112-2, LC1], 1 martillo [4112-3, LC1], mandíbula con i1 derecho y p2-m3 izquierdos [4084, LC1], porción de rama mandibular izquierda [4002-8, LC4], mandíbula con i1 izquierdo e i1-m3 derechos [4016], mandíbula con i1 izquierdo e i1, i2, p3-m3 derechos [4015, p2, p3 y p4 izquierdos con un fragmento de dentario y un trozo de arco cigomático [4107-6/7], astrágalo izquierdo [4055-18, LC1].
\end{abstract}

\section{Comentario}

Para la determinación de esta especie se tuvo en cuenta la diagnosis enmendada propuesta por Cerdeño y Bond (1998).

\section{SUBORDEN TOXODONTA Owen, 1853 \\ Familia Toxodontidae Gervais, 1847 \\ Subfamilia Xotodontinae Kraglievich, 1934 \\ Género Xotodon Ameghino, 1887}

\section{Xotodon sp.}

Fig. 31

\section{Material y procedencia}

Mitad anterior del M2 izquierdo [4019-5]. A este género pertenece probablemente un I3 izquierdo [4018].

\section{Comentario}

Este fragmento de molar es asignado al género Xotodon (= Hemixotodon) por poseer la cara labial fuertemente cóncava y la cara ántero interna muy convexa. Se distingue del holotipo de Nonotherium Castellanos porque en este último, las caras labial y mesial forman un ángulo más agudo que en Xotodon.

\section{DISCUSIÓN}

\section{EDAD}

En los últimos años se ha propuesto una serie de unidades cronoestratigráficas y bioestratigráficas para el Mioceno Tardío-Holoceno de la Región Pampeana, empleando estratotipos ubicados en el sector sur y sureste de la provincia de Buenos Aires por encontrarse allí la secuencia más completa y mejor estudiada (Cione y Tonni, 1995a, 1995b). Fuera de la región de la costa atlántica del sur de la provincia de Buenos Aires, el conocimiento que se tiene de aspectos estratigráficos, bioestratigráficos y biogeográficos del Plioceno es bastante escaso (Zanchetta, 1995), aunque existen grandes afloramientos en extensas zonas poco exploradas, especialmente en la provincia de La Rioja y Catamarca (por ejemplo, las formaciones Vinchina y Toro Negro del Terciario Superior que sobrepasan los $10.000 \mathrm{~m}$ de potencia; Ramos, 1970). El estudio del nuevo yacimiento de Las Caleras aporta valiosos datos para los temas mencionados, debido al registro de varias especies de mamíferos fósiles que nunca se habían reconocido fuera de la provincia de Buenos Aires. Estas son: Thylophorops chapalmalensis, Ringueletia simpsoni y Macroeuphractus outesi (Goin, 1995; Scillato Yané et al., 1995; Tauber et al., 1997b). La presencia de algunas de estas especies permite inferir, en forma preliminar, la edad del yacimiento de Las Caleras.

El conjunto de mamíferos registrados en el yacimiento de Las Caleras abarcan edades que van desde el Plioceno Temprano (Montehermosense) al Plioceno Tardío; no obstante, las especies Thylophorops chapalmalensis (LC1, estrato 11, Fig. 1) y Macroeuphractus outesi (LC4) son exclusivamente del Piso/Edad Chapadmalalense, según los datos de Cione y Tonni (1995a); en trabajos posteriores, el último registro de la primera especie es referido al Piso/Edad Barrancalobense (Cione y Tonni, 1995b). En el caso de Dankomys simpsoni (LC1, estrato 7, Fig. 1), se lo registró exclusivamente en el Chapadmalalense Superior (Pardiñas, 1995); en cuanto a Dolichotis sp. (LC1, estrato 1 a 11, Fig. 1 y LC4), se lo registró con dudas también a partir del Chapadmalalense Superior hasta el Lujanense, en el área de los acantilados en la costa atlántica del sur de la provincia de Buenos Aires. Con respecto al género Cardiatherium (LC4), se registra desde el Mioceno Tardío ("Edad Mamífero Huayqueriense", e.g. C. isseli), y la especie C. talicei es Montehermosense y Chapadmalalense con dudas (Mones, 1991). Este último registro se refiere a especímenes sensiblemente más pequeños que el holotipo de C. talicei (Mones, 1991); lo mismo sucede con los restos procedentes de Las Caleras, siendo probable que pertenezcan a una nueva especie.

En síntesis, esto indica que el relleno sedimentario del yacimiento de Las Caleras se depositó durante la parte media del Plioceno (Edad Chapadmalalense) y probablemente Chapadmalalense Superior, al menos los niveles prospectados. 


\section{GEOLOGÍA-TECTÓNICA}

Entre los factores que influyen en el desarrollo del ciclo cárstico, se encuentran el clima, la topografía local, la altitud relativa del macizo sobre los terrenos circundantes y el nivel de base local. Si bien no hay datos concretos sobre la edad de niveles más bajos de este sistema cárstico incipiente, se propone como hipótesis que el proceso de carstificación habría comenzado durante el Plioceno Temprano como producto de la fase tectónica temprana del MiocenoPlioceno (Pastore, 1932; Massabie y Szlafsztein, 1991; Kraemer et al., 1993).

Durante esta fase tectónica se produjo una gran actividad geológica y el bloque de la sierra Blanca habría adquirido la posición topográfica positiva necesaria para el desarrollo de este proceso de disolución. Coincidentemente se produjeron otros eventos geológicos de importancia regional, delineándose los grandes valles de las Sierras Pampeanas de Córdoba, como el valle de San Alberto (Castellanos, 1942; Sayago, 1979; Kraemer et al., 1993), valle de Punilla (De La Fuente, 1986, 1992), valle de Los Reartes (Castellanos, $1936,1944,1958)$ y otros valles menores, como el de Alpa Corral (Giannoncelli y Tauber, 1997). Además, se produjo el vulcanismo del departamento Pocho, provincia de Córdoba, entre los 7,9 y 4,5 Ma (Kay y Gordillo, 1994) y se depositaron los travertinos de la Formación La Playa (Kraglievich y Reig, 1954; Bondesio y Pascual, 1981; Donadío, 1982). Durante la edad Chapadmalalense se produjo el relleno sedimentario de las galerías, diaclasas y cavidades y la acumulación de restos de vertebrados fósiles. El proceso de carstificación culminó en tiempos postchapadmalalenses y muy probablemente en el Plioceno Tardío, como consecuencia de los movimientos tectónicos documentados por Kraemer et al. (1993) en el Valle de San Alberto. Este nuevo ascenso del bloque de la sierra Blanca provocó un cambio del nivel de base de la red fluvial local. En efecto, al descender el nivel hidrostático del bloque carstificado de la sierra Blanca, alcanzó un nuevo nivel de base, y sobre este desaguó toda la red subterránea abandonando los niveles de galerías superiores, las que se obstruyeron por hundimientos del techo de las grutas, formándose estalagmitas, estalactitas, columnas y otras estructuras por precipitación de los carbonatos, por ejemplo la costra calcárea de la galería del sitio 2 .

El bloque de la sierra Blanca desciende gradualmente hacia el sur y hacia el este, donde se halla cubierto por sedimentos del Neógeno y Cuaternario, por lo que se infiere la alta probabilidad de encontrarse más cuerpos de relleno cárstico y eventualmente restos de vertebrados fósiles, también en niveles más recientes.

\section{PALEOECOLOGÍA}

Uno de los factores más importantes que influyen en el desarrollo del ciclo cárstico es el clima, siendo mayor la carstificación bajo condiciones más cálidas y húmedas. La asociación de vertebrados permite verificar que las condiciones climáticas en general fueron templadocálidas, debido a la presencia de Tupinambis cf. teguixin, probablemente Lutreolina sp., Thylophorops chapalmalensis, Doellotatus chapadmalensis, Ringueletia simpsoni, Macroeuphractus outesi, Eumysops sp.,
Tremacyllus impressus y probablemente Xotodon sp. (Donadío, 1982; Goin, 1995; Scillato Yané et al., 1995; Vucetich y Verzi, 1995; Bond et al., 1995). En cuanto a la humedad, en general, la asociación de vertebrados no es muy indicativa, por no conocerse las exigencias de la mayoría de las especies. No obstante, es preciso aclarar que Cardiatherium, como otros Hydrochoeridae, ha sido considerado como un indicador de condiciones ambientales húmedas por el hábito de vida relacionado con cuerpos de agua estancada o de baja energía (Bondesio y Pascual, 1981); lo mismo que sucede con Lutreolina sp., que, además, es un mamífero con excelentes aptitudes para nadar. Contrariamente a esto, las especies de Dolichotis son consideradas, en general, como indicadoras de climas áridos y semiáridos (e.g. Vucetich y Verzi, 1995). Hasta el presente no se han observado evidencias que permitan inferir una mezcla de materiales, y esta aparente contradicción no estaría vinculada a un defecto del registro; por lo tanto, será preciso buscar hipótesis alternativas sobre esta asociación o sobre el significado climático de cada taxón.

Según Donadío (1982), la especie Tupinambis teguixin habita en regiones comprendidas entre las isohietas de 600 y $1600 \mathrm{~mm}$ anuales, e indicaría condiciones climáticas húmedas. Sin embargo, se han observado algunos especímenes de esta especie en cercanías de la localidad de Olta, La Rioja (Tauber, observación personal, diciembre de 1993) con un régimen pluvial inferior a los $600 \mathrm{~mm}$ anuales.

La mayoría de los taxones registrados indican un ambiente predominantemente abierto, con pastizales, o semiabierto, según las inferencias de varios autores (Goin, 1995; Scillato Yané et al., 1995; Vucetich y Verzi, 1995; Pardiñas, 1995; Bond et al., 1995). Las especies que indican ambientes abiertos son: Doellotatus chapadmalensis, Ringueletia simpsoni, Dankomys simpsoni, Eumysops sp., Lagostomus sp., Dolichotis sp. y, probablemente, Xotodon sp. La especie Tremacyllus impressus habría habitado estepas herbáceas o arbustivas.

En el caso de Macroeuphractus outesi, además de ambientes abiertos, pudo habitar áreas parcialmente arboladas (Scillato Yané et al., 1995), y la presencia de Thylophorops chapalmalensis indica la existencia de áreas semiabiertas (Goin, 1995), aunque los restos registrados de esta especie son escasos.

\section{AGRADECIMIENTOS}

El autor agradece al establecimiento minero INSUMIN S.A.C.I.F.I.M. de la provincia de Córdoba, a la Familia Maero y a la Municipalidad de la ciudad de Oncativo, por el apoyo prestado para la realización de este trabajo; el Dr. Francisco Goin contribuyó con la determinación de los didélfidos; El Sr. José Di Ronco, la Geóloga Liliana Cellenza, los estudiantes Santiago Druetta, Augusto Haro, Pablo y Claudio Tauber prestaron su inapreciable colaboración en las tareas de campo. 


\section{BIBLIOGRAFÍA}

Ameghino, F. 1883. Sobre una nueva colección de mamíferos fósiles del piso mesopotámico de la formación patagónica, recogidos en las barrancas del Paraná por el profesor Scalabrini. Boletín de la Academia Nacional de Ciencias, Córdoba, 5, 101116.

Ameghino, F. 1887. Apuntes preliminares sobre algunos mamíferos extinguidos del yacimiento de Monte Hermoso. Imprenta "El Censor", Buenos Aires, 20 pp., 2 láms.

Ameghino, F. 1888. Lista de las especies de mamíferos fósiles del Mioceno superior de Monte Hermoso, hasta ahora conocidas. Obras completas y Correspondencia científica, 5, 481-496.

Ameghino, F. 1891. Mamíferos y aves fósiles argentinos: especies nuevas. Adiciones y correcciones. Revista Argentina de Historia Natural, 1, 240-259.

Ameghino, F. 1908. Las Formaciones sedimentarias de la región litoral de Mar del Plata y Chapalmalán. Anales del Museo Nacional de Ciencias Naturales de Buenos Aires, 10, 343-428.

Bond, M., Cerdeño, E. y López, G. 1995. Los ungulados nativos de América del Sur. In: Evolución biológica y climática de la Región Pampeana durante los últimos cinco millones de años (Eds. M. T. Alberdi, G. Leone y E. P. Tonni). Madrid, 257-275.

Bondesio, P. y Pascual, R. 1981. Un Cardiatheriinae (Mammalia, Hydrochoeridae) de los calcáreos travertínicos de Las Playas (Córdoba). Edad y correlaciones. Ameghiniana, 18, 169-175.

Bordas, A. 1932. Proposición de un nuevo género para "Eutatus inornatus". Physis, 11, 167-168.

Bordas, A. 1933. Notas sobre los Eutatinae. Nueva subfamilia extinguida de Dasypodidae. Anales del Museo Nacional de Historia Natural de Buenos Aires, 37, 583-614.

Brodtkorb, M., Hillar, N. y Ametrano, S. 1981. Consideraciones genéticas en los bancos de cobre presentes en las canteras de caliza de la "Cañada de Álvarez", provincia de Córdoba. $8^{\circ}$ Congreso Geológico Argentino, San Luis, 2, 851-861.

Butler, R. F., Marshall, L. G., Drake, R. E. and Curtis, G. H. 1984. Magnetic polarity stratigraphic and ${ }^{40} \mathrm{~K}-{ }^{40} \mathrm{Ar}$ dating of late Miocene and early Pliocene continental deposits, Catamarca Province, NW Argentina. Journal of Geology, 92, 623-636.

Castellanos, A. 1936. Los sedimentos del pampeano inferior y del araucano en el valle de Los Reartes (Sierra de Córdoba). Publicaciones $N^{\circ} 6$ de la Serie Técnico Científica de la Facultad de Ciencias Matemáticas, Físico-Químicas y Naturales aplicadas a la Industria, Rosario, $110 \mathrm{pp}$.

Castellanos, A. 1942. Los sedimentos prepampeanos del valle de Nono (Sierra de Córdoba) Argentina. Publicaciones del Instituto de Fisiografía y Geología, Rosario, 13, 1-62.

Castellanos, A. 1944. Paleontología estratigráfica de los sedimentos neógenos de la provincia de Córdoba.
Publicaciones del Instituto de Fsiografía y Geología, Rosario, 23, 1-47.

Castellanos, A. 1958. Nota preliminar sobre nuevos restos de mamíferos fósiles del Brocherense del valle de Los Reartes (provincia de Córdoba, Argentina). 20 ${ }^{a}$ Sesión del Congreso Geológico Internacional, México, 7, 217 233.

Cei, J. M. 1993. Reptiles del noroeste, nordeste y este de la Argentina. Museo Regionale Di Scienze Naturali, Torino, Monografía 14, 949 pp.

Cerdeño, E. and Bond, M. 1998. Taxonomic revision and phylogeny of Paedotherium and Tremacyllus (Pachyrukhinae, Hegetotheriidae, Notoungulata) from the late Miocene to the Pleistocene of Argentina. Journal of Vertebrate Paleontology, 18, 799-811.

Cione, A. L. and Tonni, E. P., 1995a. Chronostratigraphy and "Land Mammal Ages" in the Cenozoic of Southern South America: Principles, practices and the "Uquian" problem. Journal of Paleontology, 69, 135-159.

Cione, A. L. y Tonni, E. P. 1995b. Bioestratigrafía y cronología del Cenozoico superior de la Región Pampeana. In: Evolución biológica y climática de la Región Pampeana durante los últimos cinco millones de años (Eds. M. T. Alberdi, G. Leone y E. P. Tonni). Madrid, 47-74.

Daudin, F. M. 1802. Histoire génerale et particulière des Reptiles. Paris, Impr. F. Dufart, 452 pp.

De La Fuente, M. S. 1986. Un quélido montehermosense (Plioceno Temprano-Medio) en las proximidades de Cosquín, provincia de Córdoba, Argentina. Ameghiniana, 23, 65-74.

De La Fuente, M. S. 1992. Las tortugas Chelidae del Terciario Superior y Cuaternario del territorio argentino. Ameghiniana, 29, 211-229.

Desmarest, A. G. 1820. Mammalogie ou déscription des espèces de mammifères. 2. Agasse, Paris, viii + 277 555.

Donadío, E. O. 1982. Los lacertilios fósiles de la provincia de Córdoba (Sauria, Teiidae) y sus implicancias paleoambientales. $3^{e r}$ Congreso Argentino de Paleontología y Bioestratigrafía, Corrientes, 217-223.

Francis, J. C. y Mones, A. 1965. Sobre el hallazgo de Cardiatherium talicei n. sp. (Rodentia, Hydrochoeridae) en la playa de Kiyú, Departamento de San José, República Oriental del Uruguay. Kraglieviana, 1, 3-44.

Francis, J. C. y Mones, A. 1966. La presencia de vizcachas [Lagostomus (Lagostomopsis) spicatus (Amegh)] en la Fm. Kiyú, Dto. de San José, R. O. Del Uruguay. Kraglieviana, 22, 147-168.

Giannoncelli, R. M. y Tauber, A. 1997. Nueva localidad fosilífera del Plioceno en la provincia de Córdoba, República Argentina. Ameghiniana, 34, 119.

Goin, F. J. 1995. Los Marsupiales. In: Evolución biológica y climática de la Región Pampeana durante los últimos cinco millones de años (Eds. M. T. Alberdi, G. Leone y E. P. Tonni). Madrid, 163-179.

Kay, S. M. and Gordillo, C. E. 1994. Pocho volcanic rocks and the melting of depleted continental lithosphere above a shallowly dipling subduction zone in the 
central Andes. Contribution to Mineralogy and Petrology, 117, 25-44.

Kraemer, P. E., Tauber, A., Schmidt, C. y Ramé, G. 1993. Análisis cinemático de la "falla Nono". Evidencias de actividad neotectónica. Valle de San Alberto, provincia de Córdoba. $12^{\circ}$ Congreso Geológico Argentino y $2^{\circ}$ Congreso de Exploración de Hidrocarburos, Mendoza, 3, 277-281.

Kraglievich, L. 1934. La antigüedad pliocena de las faunas de Monte Hermoso y Chapadmalal, deducidas de su comparación con las que le precedieron y sucedieron. Imprenta "El Siglo Ilustrado", 133 pp.

Kraglievich, J. L. 1957. Revisión de los roedores extinguidos del género Eumysops Ameghino, 1888 (Nota preliminar). Ameghiniana, 1, 37-39.

Kraglievich, J. L. 1965. Speciation phylétique dans les rongeurs fossiles du genre Eumysops Amegh. (Echimyidae, Heteropsomynae). Mammalia, 29, 258267.

Kraglievich, J. L. y Reig, O. A. 1954. Un nuevo prociónido del Plioceno de Las Playas (provincia de Córdoba). Revista de la Asociación Geológica Argentina, 9, 210231.

Linares, E. y La Torre, C. O. 1978. Geocronología potasioargón de las rocas metamórficas del sector meridional de la Sierra Chica de Córdoba. $7^{\circ}$ Congreso Geológico Argentino, Neuquén, 1, 787-794.

Maero, J. L. 1994. Análisis estructural, litológico y estudio de mercado de las dolomitas de Cañada de Álvarez. Trabajo Final. Departamento de Geología, Facultad de Ciencias Exactas, Físicas y Naturales, Universidad Nacional de Córdoba, 104 pp., 9 figs. (inédito).

Marshall, L. G., Butler, R. F., Drake, R. E., Curtis, G. H. and Tedford, R. H. 1979. Calibration of Great American Interchange. Science, 204, 272-279.

Martino, R. D., Guereschi, A. y Giambastiani, M. 1997. El sinformal de la sierra Blanca: petrología y evolución estructural, sierra de Comechingones, Córdoba, Argentina. Revista de la Asociación Geológica Argentina, 52, 143-156.

Massabie, A. C. y Szalafstein, C. 1991. Condiciones geomecánicas y edad del fallamiento neotectónico de las Sierras Pampeanas Orientales, Córdoba, Argentina. Actas Asociación de Geología Aplicada a la Ingeniería, Buenos Aires, 6, 154-168.

Mones, A. 1991. Monografía de la Familia Hydrochoeridae (Mammalia: Rodentia). Courier Forschungsinstitut Senckenberg, 134, 1-235.

Pardiñas, U. F. J. 1995. Los roedores cricétidos. In: Evolución biológica y climática de la Región Pampeana durante los últimos cinco millones de años (Eds. M. T. Alberdi, G. Leone y E. P. Tonni). Madrid, 227-256.

Pascual, R. y Bondesio, P. 1985. Mamíferos terrestres del Mioceno medio-tardío de las cuencas de los ríos Colorado y Negro (Argentina): evolución ambiental. Ameghiniana, 22, 133-145.

Pasotti, P. 1961. Historia hidrogeológica de la zona anegada del río de Los Molinos (prov. de Córdoba y aledaños). Publicaciones del Instituto de Fisiografía y Geología, Rosario, 44, 1-96.
Pastore, F. 1932. Hoja 20i del Mapa Geológico de la Argentina, región oriental y media de la Sierra de Córdoba. Dirección de Minería y Geología, Buenos Aires, Boletín 36, 5-67.

Quintana Salvat, F. 1981. Fotointerpretación geológica de la zona de Cañada de Álvarez, departamento de Calamuchita, provincia de Córdoba: un sinclinal volcado. Boletín de la Asociación Geológica de Córdoba, 4, 237-241.

Ramos, V. A. 1970. Estratigrafía y estructura del Terciario en la sierra de Los Colorados (provincia de La Rioja), República Argentina. Revista de la Asociación Geológica Argentina, 25, 359-382.

Reig, O. A. 1952. Descripción previa de ungulados y marsupiales fósiles del Plioceno y del Eocuaternario argentinos. Revista del Museo Municipal de Ciencias Naturales y Tradicional de Mar del Plata, 1, 19-34.

Reig. O. A. 1958. Notas para una actualización del conocimiento de la fauna de la Formación Chapadmalal. I. Lista faunística preliminar. Acta Geológica Lilloana, 11, 241-253.

Reig, O. A. 1978. Roedores cricétidos del Plioceno superior de la provincia de Buenos Aires (Argentina). Publicaciones del Museo Municipal de Ciencias Naturales de Mar del Plata "Lorenzo Scaglia”, 2, 164190.

Reig, O. A. 1994. New species of Akodontinae and Scapteromyne rodents (Cricetidae) and new records of Bolomys (Akodontini) from the upper Pliocene and middle Pleistocene of Buenos Aires province, Argentina. Ameghiniana, 31, 99-113.

Rovereto, C. 1914. Los estratos araucanos y sus fósiles. Anales del Museo Nacional de Historia Natural de Buenos Aires, 25, 1-250.

Sayago, J. M. 1979. Geomorfología del valle de "San Alberto", provincia de Córdoba. $7^{\circ}$ Congreso Geológico Argentino, Neuquén, 2, 89-107.

Scillato Yané, G. J. 1975. Presencia de Macroeuphractus retusus (Xenarthra, Dasypodidae) en el Plioceno del área Mesopotamia (Argentina). Su importancia biogeográfica y paleobiogeográfica. Ameghiniana, 12, 322-328.

Scillato Yané, G. J., Carlini, A. A., Vizcaíno, S. F. y Ortiz Jaureguizar, E. 1995. Los Xenarthros. In: Evolución biológica y climática de la Región Pampeana durante los últimos cinco millones de años (Eds. M. T. Alberdi, G. Leone y E. P. Tonni). Madrid, 181-209.

Tauber, A. A., Cellenza, L. y Di Ronco, J. 1997a. Aspectos tafonómicos del yacimiento fosilífero plioceno de Las Caleras, Departamento Calamuchita, Córdoba, República Argentina. Reunión anual de comunicaciones de la Asociación Paleontológica Argentina (Córdoba, 22-23 de noviembre de 1996), Ameghiniana, 34, 125-126.

Tauber, A. A., Hünicken, M. A. y Pensa, M. V. 1997b. Un singular yacimiento fosilífero del Plioceno de Las Caleras, departamento Calamuchita, Córdoba, Argentina. Ameghiniana, 34, 126.

Thomas, O. 1910. A collection of mammals from eastern Buenos Ayres, with descriptions of related new 
mammals from other localities. Annals and Magazine of Natural History, 8, 239-247. London.

Vucetich, M. G. y Verzi, D. H. 1995. Los roedores caviomorphos. In: Evolución biológica y climática de la Región Pampeana durante los últimos cinco millones de años (Eds. M. T. Alberdi, G. Leone y E. P. Tonni). Madrid, 211-225.
Zanchetta, G. 1995. Estado actual de la geología y estratigrafía de los depósitos plio-pleistocenos de la Región Bonaerense. In: Evolución biológica y climática de la Región Pampeana durante los últimos cinco millones de años (Eds. M. T. Alberdi, G. Leone y E. P. Tonni). Madrid, 27-45.

Manuscrito recibido: 5 de noviembre, 1999 Manuscrito aceptado: 22 de junio, 2000 